Vol. 7, No. 1, 2017

\title{
APPLICATION OF THE FREQUENCY SYMBOLIC METHOD FOR THE ANALYSIS OF A QUADRATURE DEMODULATOR OF A TELECOMMUNICATION SYSTEM
}

\author{
Bohdan Mandziy, Ksenia Chaban, Yuriy Shapovalov, Dariya Bachyk \\ Lviv Polytechnic National University, Ukraine \\ bmandziy@polynet.lviv.ua,shapov@polynet.lviv.ua,dariya.bachyk.smal@gmail.com
}

(C) Mandziy B., Chaban K., Shapovalov Yu., Bachyk D., 2017

\begin{abstract}
The paper presents the analysis of the operation of a quadrature demodulator of a signals modulated with the use of Quadrature Phase Shift Keying (QPSK) taking into account the influence of parameters of a low-pass filter. Such quadrature demodulators are used in modern telecommunication systems because of the problem of separating out two Phase Shift Keying (PSK) modulated signals (in-phase and quadrature) from the QPSK-modulated signal arising at the receiving end, which is followed by detecting and determining the binary signal as "one" or "zero". This task is performed by a two-channel demodulator of the receiver separately for in-phase and quadrature components of the QPSK-modulated signal. A wrong decision can be made due to the influence of the parasitic signal components, which occur at the inputs of decision devices. In the environment of the system UDF MAOPCs (User-Defined Functions Multivariate Analysis and Optimization of the Parametric Circuits) the influence of parameters of input signals and parameters of the synchronous detector on the output signals of the in-phase channel was analyzed. On the example of the synchronous detector, it is shown that application of the frequency symbolic method makes it possible to determine the effect of selected parameters of the circuit elements on the characteristics of functional units of telecommunications equipment in explicit form.
\end{abstract}

Key words: frequency symbolic method, UDF MAOPCs system, linear periodically-time-variable circuits, quadrature demodulator.

\section{Introduction}

In modern telecommunication systems the multipoint methods of digital modulation of carrier motion are widely used, and their advantage in comparison with binary shift keying is the smaller bandwidth of the transmission of a communication channel which is necessary for the transmission of the modulated signal [1].

In practice the multipoint phase-shift keying, which is stable in relation to the amplitude changes of a signal during its transmission through the communication channel, is the most often used.

A popular option of a multipoint phase-shift keying is the QPSK (Quadrature Phase Shift Keying), during which a certain pair of bits corresponds to each possible value of the phase of a modulated signal.

Quadrature signals are two-dimensional signals, whose instantaneous values can be mathematically determined by one complex number containing two components: in-phase (real) and quadrature (imaginary).

During the formation of QPSK-modulated signals at a transmitting end the flow of input information bits is divided into two subflows consisting of odd and even bits respectively.

Each of the subflows is used to conduct a binary phase-shift keying (PSK) of a respectively in-phase and quadrature components of carrying oscillation. After both components are added, we obtain the QPSKmodulated signal.

At the receiving end, the problem of separating out the QPSK-modulated signal into two PSK-modulated (in-phase and quadrature) signals arises followed by detecting and determining a binary signal ("one" or "zero").

This task is performed by a two-channel demodulator of the receiver separately for in-phase and quadrature components of the QPSK-modulated signal. The wrong decision can be made due to the influence of the parasitic signal components which occur at the inputs of the decision devices.

The paper presents the analysis of the operation of a quadrature demodulator of the QPSK-modulated signals taking into account the influence of the parameters of a low-pass filter.

2. Model of the quadrature demodulator and its analysis

Fig. 1 shows the flow chart of a quadrature demodulator, where the following notations are used:

$x(t)$ is an input QPSK-modulated signal; $x_{s}(t)$ is a signal obtained as the result of the detection of an in- 
phase component of the QPSK-modulated signal; $x_{k}(t)$ is a signal obtained as the result of the detection of a quadrature component of a QPSK-modulated signal;
$\mathrm{G}$ is a carrier frequency generator; $\mathrm{SD}$ is a synchronous detector (multiplier); $\mathrm{F}$ is a low-pass filter; $\mathrm{D}$ is a decision device (resolver); $\mathrm{M}$ is a multiplexer.

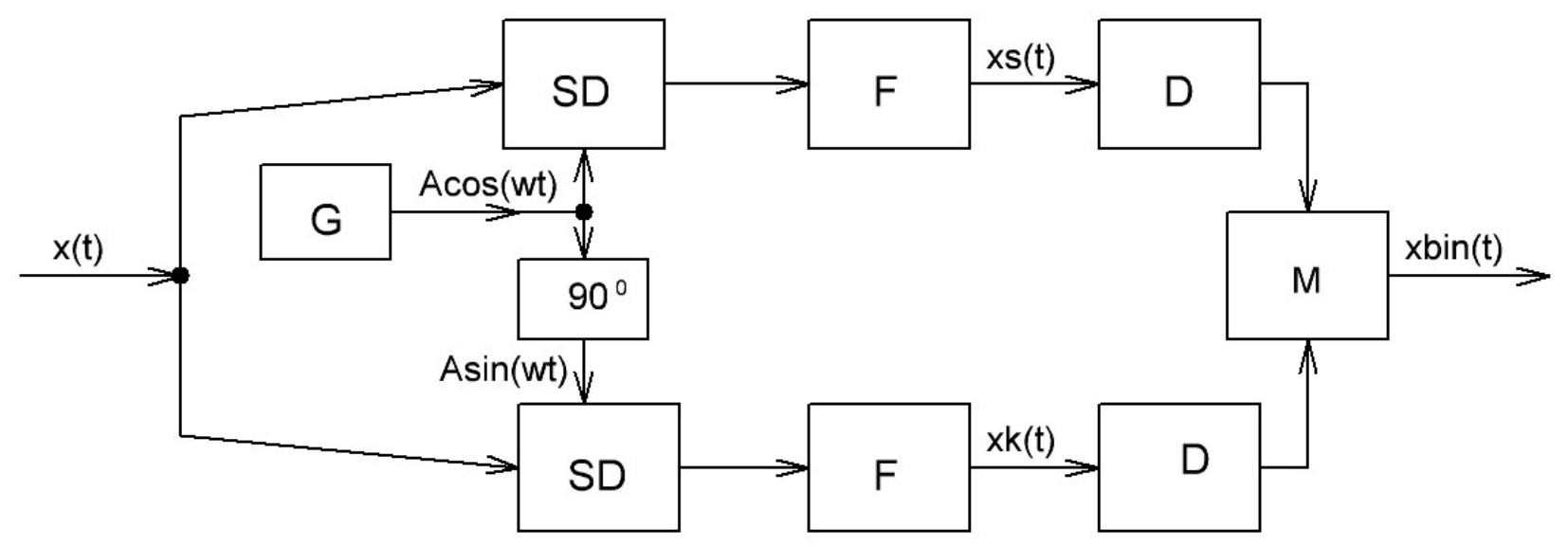

Fig. 1. Flow chart of a quadrature demodulator

The mathematical model for the k-th symbol of the input QPSK-modulated signal can be presented in the form of

$$
x_{k}(t)=X \cos \left[\omega_{n} \cdot t+\frac{(2 k-1) \pi}{4}\right],
$$

where the index $\mathrm{k}$ can take the values of $\kappa=1,2,3,4$.

Using the known trigonometric identities, expression (1) can be represented as the sum of in-phase and quadrature components of the QPSK-modulated signal respectively:

$$
\begin{aligned}
& x_{k}(t)=X \cos \left[\frac{(2 k-1) \pi}{4}\right] \cos \left(\omega_{n} \cdot t\right)- \\
& -X \sin \left[\frac{(2 k-1) \pi}{4}\right] \sin \left(\omega_{n} \cdot t\right)
\end{aligned}
$$

During the demodulation of the QPSK-modulated signal the generator frequency $G$ should be synchronized with carrier frequency $\left(\omega=\omega_{n}\right)$. Synchronous detectors SD are parametric multipliers multiplying the input QPSK-modulated signal by the in-phase and quadrature components of carrying oscillation. Fig. 2 shows the equivalent circuit of the parametric multiplier with a simple $R C$-filter, where the following notations are used: $x(t)$ is the voltage of an input QPSK-modulated signal; $i(t)$ is the source of parametric current controlled by voltage $x(t) ; C, R$ are the parameters of filter elements. In general, the filter can be of any complexity.

The dependence of the current of the controlled source on the input voltage is described by a formula:

$$
i(t)=B(t) \cdot x(t)
$$

where $\quad B(t)=B_{0}\left(1+m \cdot \cos \left(\omega_{n} \cdot t\right)\right), \quad B_{0} \quad$ is $\quad$ a coefficient characterizing the sensitivity of the current source to the changes of controlling voltage; $m$ is the coefficient of modulation depth of a parametric circuit $(0<m<1)$

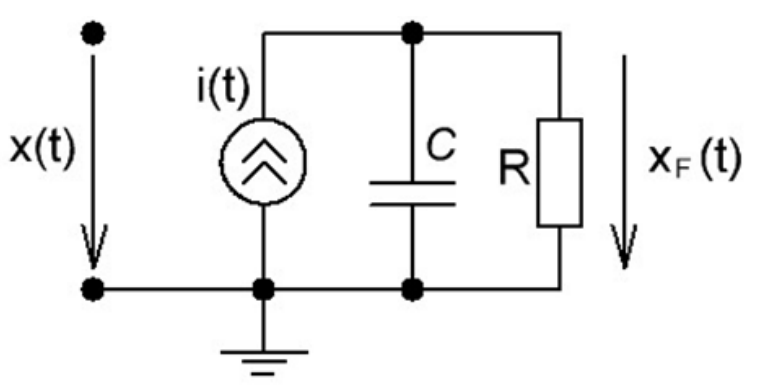

Fig. 2. Equivalent circuit of the parametric multiplier with a $R C$-filter.

The spectral structure of voltage at the outputs of the low-pass filters of the quadrature demodulator will be determined with the help of a frequency symbolic method (FS-method) [2, 3], which is based on L. A. Zadeh's method [4] allowing us to switch over from the differential equations of a parametric circuit, functioning in the time domain, to the equation describing a circuit in the frequency domain and allows us to obtain a solution in a symbolic form. The FS-method is implemented through the software in the system of UDF MAOPCs [5, $6]$, which combines the advantages of numerical and symbolic methods of circuit analysis. In our case the differential equation of the circuit in Fig.. 1 is as follows:

$$
R \cdot C \cdot \frac{d x_{F}(t)}{d t}+x_{F}(t)=R \cdot B(t) \cdot x(t)
$$


From expression (4) and according to [2], we obtain the equation describing the given circuit in the frequency domain $(1 / R=Y)$ :

$$
C \cdot W^{\prime}(s, t)+(Y+C \cdot s) \cdot W(s, t)=B(t)
$$

where

$$
W(s, t)=\frac{x_{F}(s, t)}{x(s)} \text { is a conjugate parametric }
$$

transfer function, which is equal to the relation of the depiction of an output signal of the synchronous detector $X_{F}(s, t)$ to the depiction of its input signal $X(s, t)$; $s$ is complex frequency. It should be noted that for the parametric circuit the conjugate parametric transfer function depends simultaneously both on time and on frequency, while in case of the circuit with constant parameters, the complex transfer function depends only on the frequency.

The conjugate parametric transfer function $W(s, t)$ will be approximated by one harmonic component:

$$
\begin{aligned}
W(s, t) \approx & W_{0}(s)+W_{c 1}(s) \cos (\omega t)+ \\
& +W_{s 1}(s) \sin (\omega t) .
\end{aligned}
$$

As a result we obtain the symbolic expressions of the conjugate parametric transfer functions:

A) for the in-phase channel:

$$
\begin{aligned}
& W_{s}(s, t)=\frac{B_{0}}{j \cdot C \cdot \omega+Y}+ \\
& +\frac{m \cdot B_{0}\left[\left(1+j \cdot \omega \cdot C \cdot \frac{1}{Y}\right) \cdot \cos (\omega t)\right]}{2 \cdot j \cdot C \cdot \omega+Y}+ \\
& +\frac{m \cdot B_{0}\left[\omega \cdot C \cdot \frac{1}{Y} \cdot \sin (\omega t)\right]}{2 \cdot j \cdot C \cdot \omega+Y}
\end{aligned}
$$

B) for the quadrature channel:

$$
\begin{aligned}
& W_{k}(s, t)=\frac{B_{0}}{j \cdot C \cdot \omega+Y}+ \\
& +\frac{m \cdot B_{0}\left[\left(1+j \cdot \omega \cdot C \cdot \frac{1}{Y}\right) \cdot \sin (\omega t)\right]}{2 \cdot j \cdot C \cdot \omega+Y}- \\
& -\frac{m \cdot B_{0}\left[\omega \cdot C \cdot \frac{1}{Y} \cdot \cos (\omega t)\right]}{2 \cdot j \cdot C \cdot \omega+Y} .
\end{aligned}
$$

The complex amplitudes of the output voltages of the low-pass filters in the synchronous detectors equal:
A) for the in-phase channel:

$$
\begin{aligned}
& X_{s}=\frac{j \cdot X \cdot \exp (j \cdot \varphi) \cdot m \cdot B_{0} \cdot C \cdot \omega}{\left(2 \cdot j \cdot C \cdot \omega \cdot Y+Y^{2}\right)}+ \\
& +\frac{X \cdot \exp (j \cdot \varphi) \cdot m \cdot B_{0} \cdot Y}{2 \cdot\left(2 \cdot j \cdot C \cdot \omega \cdot Y+Y^{2}\right)}+ \\
& +\frac{X \cdot \exp (j \cdot \omega t)^{2} \cdot \exp (j \cdot \varphi) \cdot m \cdot B_{0} \cdot Y}{2 \cdot\left(2 \cdot j \cdot C \cdot \omega \cdot Y+Y^{2}\right)}+ \\
& +\frac{X \cdot \exp (j \cdot \omega t) \cdot \exp (j \cdot \varphi) \cdot B_{0}}{(j \cdot C \cdot \omega+Y)}
\end{aligned}
$$

B) for the quadrature channel:

$$
\begin{gathered}
X_{k}=-\frac{X \exp (j \cdot \varphi) \cdot m \cdot B_{0} \cdot C \cdot \omega}{\left(2 j C \omega Y+Y^{2}\right)}+ \\
+\frac{j \cdot X \cdot \exp (j \cdot \varphi) \cdot m \cdot B_{0} \cdot Y}{2 \cdot\left(2 \cdot j \cdot C \cdot \omega \cdot Y+Y^{2}\right)}- \\
-\frac{j \cdot X \cdot \exp (j \cdot \omega \cdot t)^{2} \cdot \exp (j \cdot \varphi) \cdot m \cdot B_{0} \cdot Y}{2 \cdot\left(2 \cdot j \cdot C \cdot \omega \cdot Y+Y^{2}\right)}+ \\
+\frac{X \exp (j \cdot \omega t) \cdot \exp (j \cdot \varphi) \cdot B_{0}}{(j C \omega+Y)} .
\end{gathered}
$$

The first terms of expressions (9) and (10) are useful low-frequency components that pass through the RCfilters and are used for determining the phase $\varphi$ of the input modulated signal. The second and third terms in these expressions describe high-frequency parasitic components of output signals with frequencies $\omega$ and $2 \cdot \omega$ respectively.

\section{Computer experiment}

In the UDF MAOPCs environment, using expression (9), the analysis of the influence of the parameters of input signals and parameters of the synchronous detector on the output signals of the in-phase channel was conducted.

Fig. 3 presents a fragment of the program code in the UDF MAOPCs environment corresponding to the conducted computer experiment.

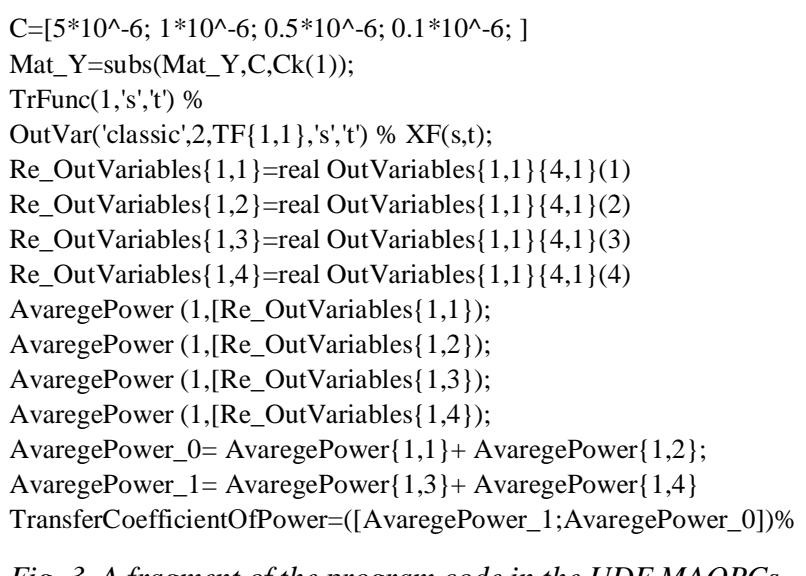

Fig. 3. A fragment of the program code in the UDF MAOPCs 
Fig. 4 shows time dependences of the output voltage of the in-phase channel that were calculated for such

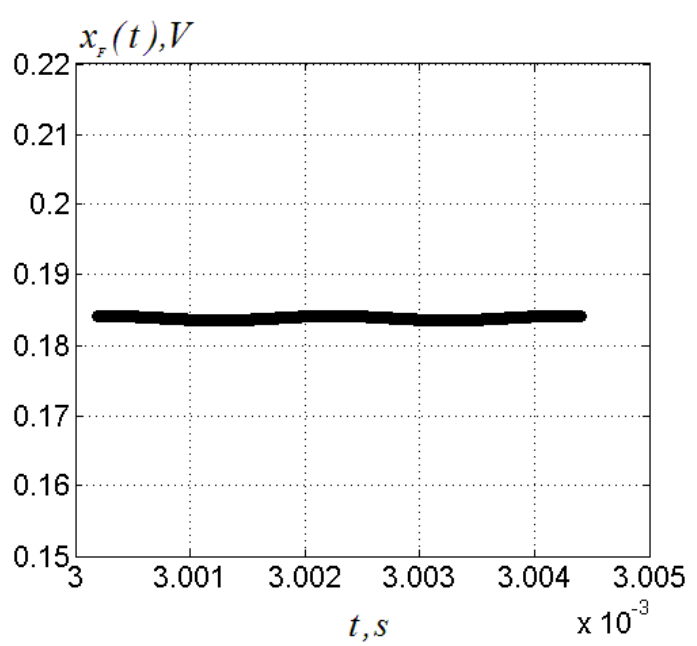

a) $C=5 \mu F$

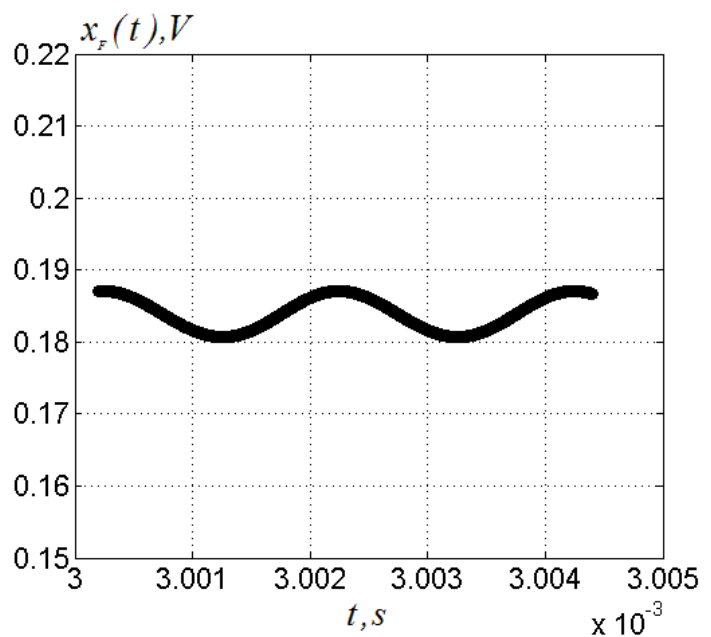

c) $C=0.5 \mu F$

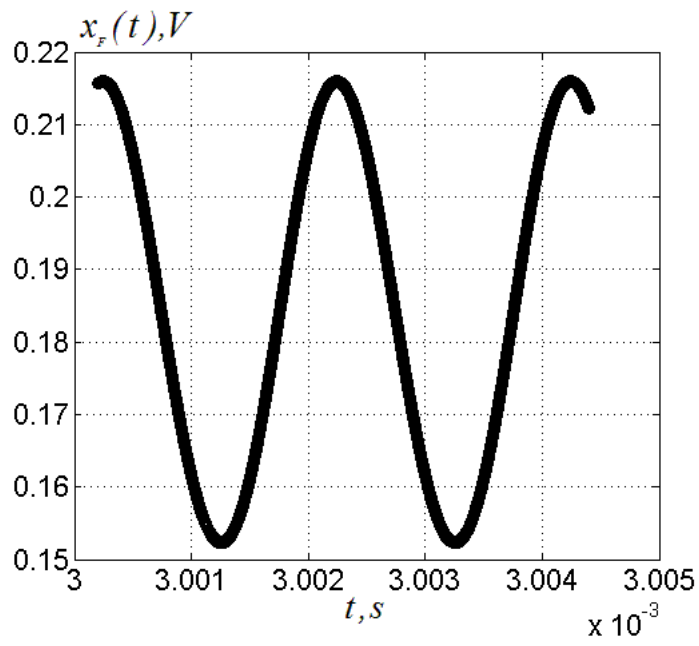

e) $C=0.05 \mu F$; numerical parameters: $\quad B_{0}=50 \mathrm{~mA} / \mathrm{V}, \mathrm{m}=0.052$, $Y=5 \cdot 10^{-4} S, \varphi=\pi / 4, \omega=2 \pi 500 \cdot 10^{3} \mathrm{rad} / \mathrm{s}, X=0.1 \mathrm{~V}$.

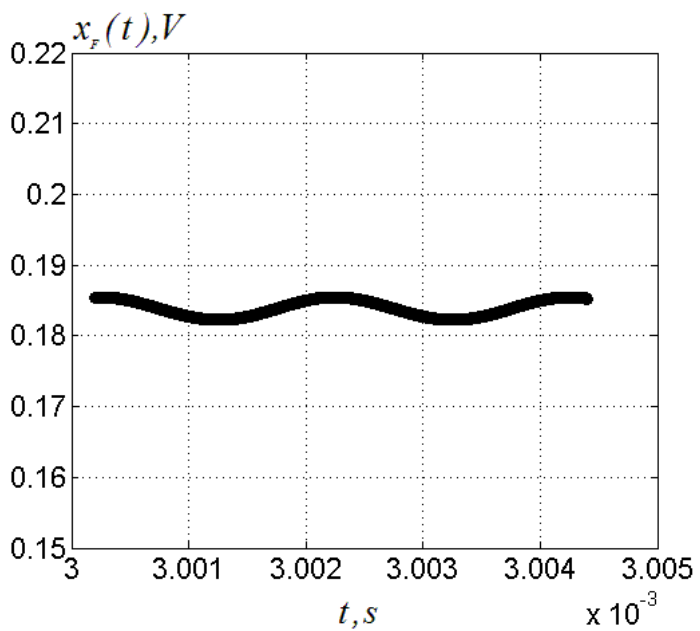

b) $C=1 \mu F$

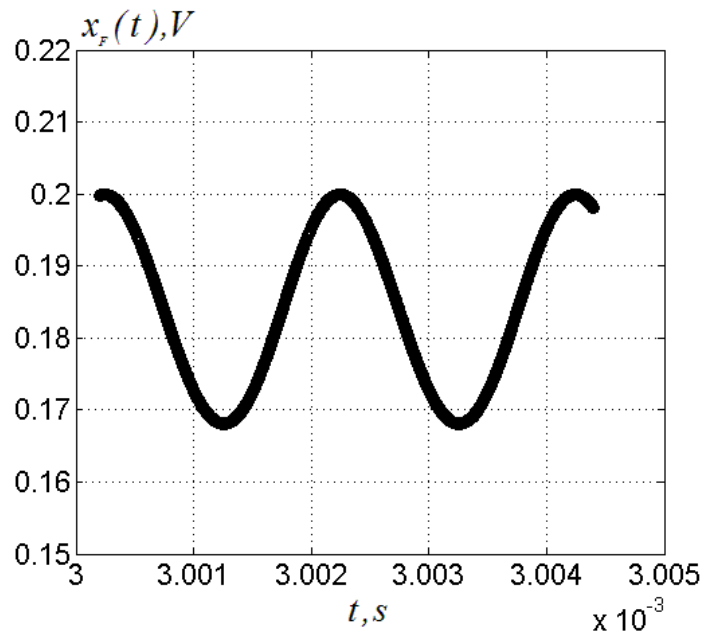

d) $C=0.1 \mu F$;

Fig. 4. The time dependences of the output voltage of the inphase channel of the quadrature synchronous detector for different values of capacitance of low-pass filter. 
Table 1 shows the values of average powers:

$P_{0}=\left(\operatorname{Re}\left[\begin{array}{l}\frac{j \cdot X \cdot \exp (j \cdot \varphi) \cdot m \cdot B_{0} \cdot C \cdot \omega}{\left(2 \cdot j \cdot C \cdot \omega \cdot Y+Y^{2}\right)} \\ +\frac{X \cdot \exp (j \cdot \varphi) \cdot m \cdot B_{0} \cdot Y}{2\left(2 \cdot j \cdot C \cdot \omega \cdot Y+Y^{2}\right)}\end{array}\right]\right)^{2}-\quad$ is the average power of the useful low-frequency component,

$P_{a v 1}=\frac{1}{T} \int_{0}^{T}\left(\operatorname{Re} \frac{X \cdot \exp (j \omega t)^{2} \cdot \exp (j \varphi) \cdot m \cdot B_{0} \cdot Y}{2\left(2 \cdot j \cdot C \cdot \omega Y+Y^{2}\right)}\right)^{2} d t$ and

$P_{a v 2}=\frac{1}{T} \int_{0}^{T}\left(\operatorname{Re} \frac{X \cdot \exp (j \cdot \omega \cdot t) \cdot \exp (j \cdot \varphi) \cdot B_{0}}{(j \cdot C \cdot \omega+Y)}\right)^{2} d t-$ are the average powers of parasitic high-frequency components of output signals with frequencies $\omega$ and $2 \omega$, consequently, for different values $C$ and phase $\varphi$ of the input signal.

Values of average powers

Table 1

\begin{tabular}{|c|c|c|c|}
\hline \multicolumn{4}{|c|}{$\varphi=45^{\circ}$} \\
\hline$C$ & $P_{0}$ & $P_{a v 1}$ & $P_{a v 2}$ \\
\hline $5 \mu F$ & 0.0338 & $0.8561 \mathrm{e}-11$ & $0.5066 \mathrm{e}-7$ \\
\hline $1 \mu F$ & 0.0338 & $0.2140 \mathrm{e}-9$ & $0.1266 \mathrm{e}-5$ \\
\hline $0.5 \mu F$ & 0.0338 & $0.8561 \mathrm{e}-9$ & $0.5066 \mathrm{e}-5$ \\
\hline $0.1 \mu F$ & 0.0338 & $0.2140 \mathrm{e}-7$ & $0.1266 \mathrm{e}-3$ \\
\hline $0.05 \mu F$ & 0.0338 & $0.8562 \mathrm{e}-7$ & $0.5066 \mathrm{e}-3$ \\
\hline \multicolumn{4}{|c|}{$\varphi=135^{\circ}$} \\
\hline$C$ & $P_{0}$ & $P_{a v 1}$ & $P_{a v 2}$ \\
\hline $5 \mu F$ & 0.0338 & $0.8561 \mathrm{e}-11$ & $0.5066 \mathrm{e}-7$ \\
\hline $1 \mu F$ & 0.0338 & $0.2140 \mathrm{e}-9$ & $0.1266 \mathrm{e}-5$ \\
\hline $0.5 \mu F$ & 0.0338 & $0.8561 \mathrm{e}-9$ & $0.5066 \mathrm{e}-5$ \\
\hline $0.1 \mu F$ & 0.0338 & $0.2140 \mathrm{e}-7$ & $0.1266 \mathrm{e}-3$ \\
\hline $0.05 \mu F$ & 0.0338 & $0.8562 \mathrm{e}-7$ & $0.5066 \mathrm{e}-3$ \\
\hline \multicolumn{4}{|c|}{$\varphi=225^{\circ}$} \\
\hline$C$ & $P_{0}$ & $P_{a v 1}$ & $P_{a v 2}$ \\
\hline $5 \mu F$ & 0.0338 & $0.8561 \mathrm{e}-11$ & $0.5066 \mathrm{e}-7$ \\
\hline $1 \mu F$ & 0.0338 & $0.2140 \mathrm{e}-9$ & $0.1266 \mathrm{e}-5$ \\
\hline $0.5 \mu F$ & 0.0338 & $0.8561 \mathrm{e}-9$ & $0.5066 \mathrm{e}-5$ \\
\hline $0.1 \mu F$ & 0.0338 & $0.2140 \mathrm{e}-7$ & $0.1266 \mathrm{e}-3$ \\
\hline $0.05 \mu F$ & 0.0338 & $0.8562 \mathrm{e}-7$ & $0.5066 \mathrm{e}-3$ \\
\hline \multicolumn{4}{|c|}{$\varphi=315^{\circ}$} \\
\hline$C$ & $P_{0}$ & $P_{a v 1}$ & $P_{a v 2}$ \\
\hline $5 \mu F$ & 0.0338 & $0.8561 \mathrm{e}-11$ & $0.5066 \mathrm{e}-7$ \\
\hline $1 \mu F$ & 0.0338 & $0.2140 \mathrm{e}-9$ & $0.1266 \mathrm{e}-5$ \\
\hline $0.5 \mu F$ & 0.0338 & $0.8561 \mathrm{e}-9$ & $0.5066 \mathrm{e}-5$ \\
\hline $0.1 \mu F$ & 0.0338 & $0.2140 \mathrm{e}-7$ & $0.1266 \mathrm{e}-3$ \\
\hline $0.05 \mu F$ & 0.0338 & $0.8562 \mathrm{e}-7$ & $0.5066 \mathrm{e}-3$ \\
\hline
\end{tabular}

Table 2 shows obtained values of the ratio of the sum of average powers of parasitic components to the average power of useful component for different values $C$ and phase $\varphi$ of the input signal.

Table 2

\begin{tabular}{|c|c|c|c|c|}
\hline \multicolumn{5}{|c|}{$\alpha=\frac{\left(P_{a v 1}+P_{a v 2}\right)}{P_{0}}$} \\
\hline$C$ & $\varphi=45^{\circ}$ & $\varphi=135^{\circ}$ & $\varphi=225^{\circ}$ & $\varphi=315^{\circ}$ \\
\hline $5 \mu F$ & $0.1499 \mathrm{e}-5$ & $0.1499 \mathrm{e}-5$ & $0.1499 \mathrm{e}-5$ & $0.1499 \mathrm{e}-5$ \\
\hline $1 \mu F$ & $0.3747 \mathrm{e}-4$ & $0.3747 \mathrm{e}-4$ & $0.3747 \mathrm{e}-4$ & $0.3747 \mathrm{e}-4$ \\
\hline $0.5 \mu F$ & $0.1499 \mathrm{e}-3$ & $0.1499 \mathrm{e}-3$ & $0.1499 \mathrm{e}-3$ & $0.1499 \mathrm{e}-3$ \\
\hline $0.1 \mu F$ & $0.3747 \mathrm{e}-2$ & $0.3747 \mathrm{e}-2$ & $0.3747 \mathrm{e}-2$ & $0.3747 \mathrm{e}-2$ \\
\hline $0.05 \mu F$ & $0.1499 \mathrm{e}-1$ & $0.1499 \mathrm{e}-1$ & $0.1499 \mathrm{e}-1$ & $0.1499 \mathrm{e}-1$ \\
\hline
\end{tabular}

\section{Conclusions}

1. On the example of the synchronous detector it is shown that the application of the frequency symbolic method makes it possible to determine in an explicit form the effect of selected parameters of the circuit elements on the characteristics of functional units of telecommunications equipment.

2. Analytical expressions obtained in this paper can be used for calculating the signal / noise ratio at the output of synchronous detector and assessing the missing error probability in the device.

3. The conducted computer experiments confirmed the computation efficiency of the symbolic analysis in the environment of the UDF MAOPCs system, as the calculation of one variant of the circuit takes 5 seconds, allowing us to analyze complicated circuits of multipliers of signals and filters.

\section{References}

[1] P. Vanassche, G. Gielen, and W. Sansen, Systematic Modeling and Analysis of Telecom Frontends and their Building Blocks. Springer; 2005.

[2] Yu. Shapovalov, B. Mandziy, and S. Mankovsky, "The peculiarities of analysis of linear parametric circuit performed by frequency-symbolic method", Przeglad Elektrotechniczny, vol. 86, no. 1, pp. 158160, 2010.

[3] Yu. Shapovalov, Symbolic analysis of linear electrical circuits in the frequency domain. Fixed and variable parameters. Lviv, Ukraine: Lviv Polytechnic National University, 2014.

[4] L. A. Zadeh, "Frequency Analysis of Variable Networks", Proc. IRE, vol. 38, no. 3, pp. 291-299, 1950 . 
[5] Yu. Shapovalov, B. Mandziy, and D. Bachyk, "The system functions MAOPCs for analysis and optimization of linear periodically time-variable circuits based on the frequency symbolic method", Przeglad Elektrotechniczny, vol. 91, no. 7, pp. 3942, 2015.

[6] Yu. Shapovalov, B. Mandziy, and D.Bachyk, "The determination of power of linear periodically-timevariable circuits in an environment of the system of software functions MAOPCs', Bulletin of National Technical University of Ukraine. Series Radiotechnique. Radioapparatus design, no. 60, pp. 3444, 2015.

\section{ЗАСТОСУВАННЯ ЧАСТОТНОГО СИМВОЛЬНОГО МЕТОДУ ДЛЯ АНАЛІЗУ КВАДРАТУРНОГО ДЕМОДУЛЯТОРА ТЕЛЕКОМУНІКАЦІЙНОЇ СИСТЕМИ}

Богдан Мандзій, Ксенія Чабан, Юрій Шаповалов, Дарія Бачик

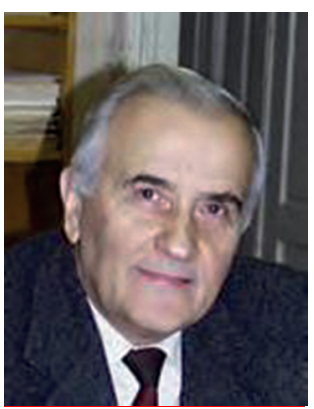

Bohdan Mandziy - Ph.D., Sc.D., professor of the Depart-ment of Theoretical Radio Engi-neering and Radio Measure-ment, Lviv Polytechnic National University, Ukraine. Author of over 250 scientific and educational works, including 2 monographs and 6 textbooks.

The research area includes methods of automated analysis of nonlinear electronic circuits and ensuring reliability of radio electronic devices and telecommunication equipment.

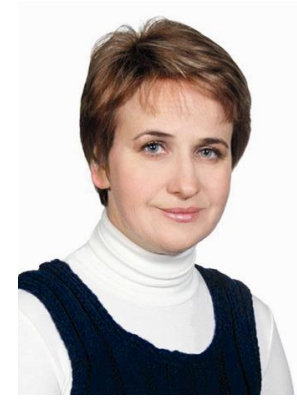

Ksenia Chaban is Associate Professor of the Department of Telecommunications of Lviv Polytechnic National University. She received her Ph.D. degree in mathematical software for computer systems and networks in 2008 from Lviv Polytechnic National University. Her research activities currently cover systems of switching and information distribution.

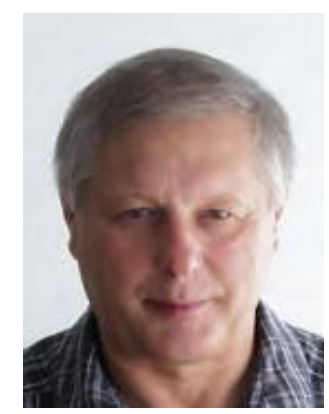

Yuriy Shapovalov - Ph.D., Sc.D., professor of the Department of Radioelectronic Devices and Systems, Lviv Polytechnic National University, Ukraine. The author of over 50 publications. His research area is frequency symbolic analysis of linear periodically time-variable (LPTV) circuits. IEEE member since 2017.

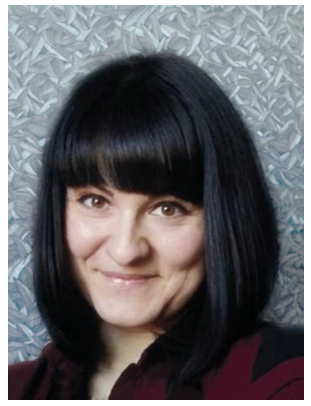

Dariya Bachyk - Ph.D., Assistant Professor of the Department of Radioelectronic Devices and Systems, Lviv Polytechnic National University, Ukraine. The author of over 20 publications. Research interests investigation of stability of LPTV circuits by frequency symbolic method, investigation of frequency symbolic models, multivariate analysis and optimization LPTV circuits. IEEE member since 2017. 